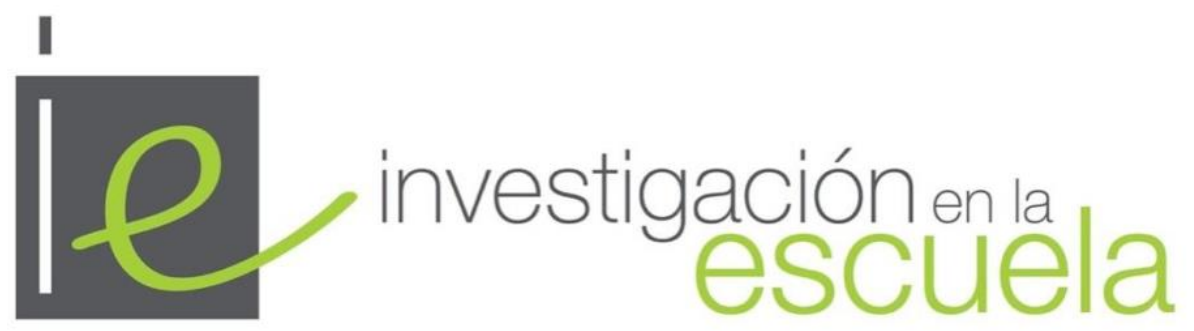

Revista de Investigación e Innovación Educativa nº 104, 2021 | e-ISSN 2443-9991

\title{
Concepciones del profesorado durante un proceso formativo en torno al trabajo por proyectos: estudio de un caso en Educación Primaria
}

Teachers' conceptions during a training process around project based work: a case study in Primary Education

Dr. José Ramón Mora Márquez es Investigador en la Facultad de Educación de la Universidad de Huelva (España) ·moramarquez91@gmail.com·https://orcid.org/0000-0002-4773-092X

iD Dr. Gabriel H. Travé González es profesor Sustituto Interino en la Facultad de Educación de la Universidad de Huelva (España)·gabriel.trave@dedu.uhu.es·https://orcid.org/0000-0002-0887-8649

Cómo citar este artículo

Mora Márquez, J. R. y Travé González, G. H. (2021). Concepciones del profesorado durante un proceso formativo en torno al trabajo por proyectos: estudio de un caso en Educación Primaria. Investigación en la Escuela, 104, 41-52. doi: http:/ /dx.doi.org/10.12795/IE.2021.i104.04

Resumen. El trabajo por proyectos constituye una alternativa curricular con potencialidad para mejorar los procesos de enseñanza-aprendizaje en los centros educativos. En el presente artículo se detalla el proceso de formación llevado a cabo por el profesorado de un centro de Educación Infantil y Primaria antes y durante la implementación de un proyecto de trabajo por proyectos. La investigación, a partir de la metodología de estudio de casos, ha utilizado métodos mixtos tanto cualitativos (a través de entrevistas, observación directa y análisis de documentos), como cuantitativos, mediante la administración de un cuestionario. Los resultados del estudio muestran que tanto el proceso formativo desarrollado como el diseño y desarrollo de un proyecto de trabajo en el centro, ha representado una oportunidad de aprendizaje para alumnado y profesorado. Sin embargo, la apuesta por el trabajo por proyectos evidencia las dificultades que experimenta el centro para su materialización práctica, especialmente en cuanto a las limitaciones temporales para el diseño y evaluación del proyecto. Las conclusiones muestran las ventajas de fomentar procesos formativos en los centros en torno al trabajo por proyectos, además de permitir extraer una serie de recomendaciones a modo de propuestas para la mejora y sostenibilidad de las experiencias.

Abstract. Project work has the potential to improve teaching and learning processes in schools. This article details the training process carried out by the teachers of an Early Childhood and Primary Education center before and during the implementation of a project work initiative. The research utilized a case study methodology using mixed methods, both qualitative (through interviews, direct observation and document analysis), and quantitative, through the administration of a questionnaire. The results of the study show that the training process, as well as the design and development of the project, have represented a learning opportunity for students and teachers. However, the commitment to project work shows an apparent difficulty in implementation, especially in terms of time limitations for its design and evaluation. In summary, the study has shown the advantages of promoting training processes within the schools in regards to project work. In addition, proposals for the improvement and sustainability of the experiences are recommended.

\section{Palabras clave $\cdot$ Keywords}

Trabajo por proyectos, concepciones, innovación, estudio de casos, formación, aprendizaje basado en proyectos (ABP).

Project based work, conceptions, innovation, case studies, teacher training, Project Based Learning (PBL). 


\section{Introducción}

Son muchas las evidencias que ponen de manifiesto la complejidad de materializar innovaciones y mejoras sostenidas en el tiempo en el seno de las escuelas (Sarason, 2003). La clave para transformar los procesos de enseñanza/aprendizaje no es simple, pero cualquier propuesta de cambio deberá priorizar el debate acerca del papel del currículum y el desarrollo profesional del profesorado (Santos Guerra, 2010; Stenhouse, 1987; Halinen, 2018).

Ante tamaño reto, la perspectiva educativa de trabajo por proyectos posibilita redefinir los procesos de enseñanza/aprendizaje que acontecen en las escuelas, al fundamentarse en propuestas cooperativas, en las que la investigación escolar, la negociación de significados, la participación democrática y la inclusión educativa son señas identitarias (Leat, 2017; Majó y Baqueró, 2014; Pozuelos, 2007). La concreción de los proyectos en los centros da lugar a una gran diversidad de propuestas que parten de problemáticas sobre las que se investiga para responder a los interrogantes a partir del desarrollo de actividades, experiencias, talleres y salidas de investigación (Sanmartí y Márquez, 2017). El conocimiento se construye de forma colaborativa, afianzando aprendizajes significativos inspirados por la conjugación de saberes y recursos diversos en contextos de participación e intercambio de ideas (Larmer \& Mergendoller, 2010).

El profesorado que se involucra en este tipo de procesos experimenta una mejora de su desarrollo profesional, desde el momento en que se generan dinámicas de trabajo colegiado en el seno de los centros a partir de procesos de diseño, desarrollo y evaluación de los proyectos (Guo \& Yang, 2012; Pozuelos et al., 2017; Travé et al., 2019). Asimismo, se documentan numerosas ventajas para el alumnado, tanto en lo referente a la compresión del contenido, el desarrollo de sus competencias y la motivación por aprender (Chen \& Yang, 2019; González-Rodríguez et al., 2020; Han et al., 2015; Kokotsaki et al., 2016).

Sin embargo, a pesar de sus bondades, a día de hoy el desarrollo de propuestas de trabajo por proyectos sigue siendo minoritario, en un contexto educativo muy marcado por el peso de la tradición disciplinar y la presencia hegemónica del libro de texto en las aulas (Soto y Travé, 2018). Las propuestas de trabajo por proyectos son percibidas con recelo por parte del profesorado, unos al considerar el aumento de carga laboral (Aksela \& Haatainen, 2019), otros al argumentar que "con esta forma de presentar el currículum muchos contenidos importantes se diluyen o no son abordados con el rigor que estos merecen" (Pozuelos y García-Prieto, 2020, p. 49). Aún más escasos son los estudios que analizan los procesos formativos desarrollados en torno a la perspectiva educativa de trabajo por proyectos en el marco de centros que diseñan y desarrollan una experiencia. Domènech-Casal et al. (2019) analizaron 87 diseños de proyectos realizados por docentes de Educación Secundaria a partir de diferentes sesiones formativas. El estudio puso de manifiesto la alta satisfacción del profesorado con acciones formativas vinculadas a la práctica real, la posibilidad de contar con mentores, el acceso a proyectos previamente aplicados en la práctica y a recursos prácticos. Además, la formación del profesorado ayuda a desmitificar ideas preconcebidas y a crear certidumbre en torno a los procesos de diseño, desarrollo y evaluación de los proyectos, eliminando barreras que limitan el éxito de las experiencias (Gutiérrez, 2015). Por ello, son necesarias investigaciones que aborden los muchos interrogantes que se generan en torno a esta temática, entre otros: ¿cómo ha sido el proceso de formación acerca del trabajo por proyectos?, ¿cuál es el grado de implicación del profesorado?, ¿Cómo se lleva a cabo la planificación?, ¿cómo es el desarrollo del proyecto?, ¿cómo son los recursos y materiales?, ¿cuál es el papel del profesorado? ¿y del alumnado? y ¿cómo se aborda la evaluación del proyecto?

Seguidamente se presenta una investigación con la finalidad de dar respuesta a estos interrogantes a partir de los siguientes objetivos:

Examinar el desarrollo de un proceso formativo en torno al trabajo por proyectos en un centro de Educación Infantil y Primaria.

Analizar las concepciones y prácticas del profesorado del centro en torno al diseño y desarrollo de un proyecto de trabajo.

\section{Material y método}

En esta investigación se ha llevado a cabo un estudio descriptivo acerca de cómo se desarrolla el trabajo por proyectos en un centro de Educación Infantil y Primaria. El método elegido ha sido el estudio de caso único donde $\mathrm{n}=1$. El caso tiene que ser algo específico y complejo y estar en funcionamiento. Se argumenta en Stake (1999) que para la elección de un caso es primordial escoger aquellos que sean sencillos de abordar y donde las indagaciones realizadas sean bien recibidas. Es por ello, que antes de abordar esta investigación, 
se contaba con informadores clave que respaldaron el estudio y la colaboración de los agentes involucrados en el caso.

Para el desarrollo del estudio se han establecido dos categorías a partir de los objetivos propuestos. Se caracterizan en la siguiente tabla conjuntamente con los contenidos, instrumentos de investigación y el proceso desarrollado para la obtención de la información.

Tabla 1

Categorías, objetivos, contenidos, instrumentos de investigación y proceso realizado en la investigación

\begin{tabular}{|c|c|c|c|c|}
\hline Categorías & Objetivos & Contenidos & $\begin{array}{l}\text { Instrumentos } \\
\text { investigación }\end{array}$ & $\begin{array}{l}\text { Proceso } \\
\text { realizado }\end{array}$ \\
\hline $\begin{array}{l}\text { 1. Representaciones } \\
\text { en torno al } \\
\text { itinerario formativo } \\
\text { sobre trabajo por } \\
\text { proyectos }\end{array}$ & $\begin{array}{l}\text { Examinar el } \\
\text { desarrollo de un } \\
\text { proceso formativo } \\
\text { en torno al trabajo } \\
\text { por proyectos en } \\
\text { un centro de } \\
\text { Educación Infantil } \\
\text { y Primaria. }\end{array}$ & $\begin{array}{l}\text {-Valoraciones del } \\
\text { proceso formativo } \\
\text {-Concepciones } \\
\text { sobre trabajo } \\
\text { proyectos }\end{array}$ & $\begin{array}{l}\text {-Entrevista } \\
\text {-Análisis de } \\
\text { documentos } \\
\text {-Observación } \\
\text { directa } \\
\text {-Diario de } \\
\text { investigación } \\
\text {-Cuestionario } \\
\text { sobre trabajo }\end{array}$ & $\begin{array}{l}\text {-Tres entrevistas a } \\
\text { docentes: tutor de } \\
3^{\circ} \mathrm{EP}, \\
\text { coordinadora } \\
\text { proyecto y jefa de } \\
\text { estudios. } \\
\text {-Entrevista grupal } \\
\text { con alumnado de } \\
3^{\circ} \mathrm{EP} \text {. }\end{array}$ \\
\hline $\begin{array}{l}\text { 2. Diseño y } \\
\text { desarrollo de un } \\
\text { proyecto de trabajo }\end{array}$ & $\begin{array}{l}\text { Analizar las } \\
\text { concepciones y } \\
\text { prácticas del } \\
\text { profesorado del } \\
\text { centro en torno al } \\
\text { diseño y desarrollo } \\
\text { de un proyecto de } \\
\text { trabajo. }\end{array}$ & $\begin{array}{l}\text { 1.Planificación del } \\
\text { proyecto (diseño: } \\
\text { red preguntas, } \\
\text { objetivos, } \\
\text { contenidos, } \\
\text { actividades, } \\
\text { evaluación, } \\
\text { recursos) } \\
\text { 2. Implementación } \\
\text { (Papel del, } \\
\text { alumnado, } \\
\text { profesorado y } \\
\text { familias) }\end{array}$ & $\begin{array}{l}\text { por proyectos } \\
\text { en el aula }\end{array}$ & $\begin{array}{l}\text {-Entrevista grupal } \\
\text { a madres } \\
\text { colaboradoras. } \\
\text {-Análisis de } \\
\text { documentos: } \\
\text { proyecto de } \\
\text { centro, página } \\
\text { web, memoria de } \\
\text { la Formación en } \\
\text { Centro. } \\
\text {-Vídeos y } \\
\text { fotografías. } \\
\text {-Redacción de un } \\
\text { diario de } \\
\text { investigación. } \\
\text {-5 observaciones } \\
\text { de aula. } \\
\text {-19 cuestionarios } \\
\text { a docentes }\end{array}$ \\
\hline
\end{tabular}

Para su desarrollo se han utilizado diferentes instrumentos y técnicas de carácter mixto, tanto cualitativas como cuantitativas. Se han establecido cuatro apartados para ordenar los instrumentos de recogida de datos y dotarlos de significado. La elección de cada uno de ellos ha sido realizada con el mayor rigor posible y para que exprese de la manera más fidedigna la realidad existente en el centro.

En primer lugar, se han llevado a cabo observaciones para contemplar la realidad que acontece en torno al proyecto. El tipo de observación ha sido directa, ya que se ha estado presente en gran parte de las actividades y en el propio desarrollo del proyecto. Asimismo, se han utilizado medios fotográficos y vídeos para corroborar lo sucedido durante todo el proceso. Se han analizado también documentos propios del centro. Es el caso, por ejemplo, del proyecto educativo, el cual ayudó a conocer el contexto sociocultural de las familias y la idiosincrasia del propio centro. También han sido analizados otros documentos aportados por la asesoría de formación como la memoria de seguimiento y memoria final. Dichos documentos fueron complementados por el profesorado a lo largo del proceso formativo y documentan los logros alcanzados, las dificultades y las oportunidades de mejora. Se ha asignado un número al azar a cada docente participante con el fin de identificar sus aportaciones. Para extraer todo el jugo del proyecto y de lo que ocurre alrededor del mismo, hay que vivir en primera persona su desarrollo. Asimismo, se recopila la información 
pormenorizada utilizando el diario del investigador, con el cual podemos tomar notas, hacer dibujos o apuntar cualquier hecho que esté ocurriendo en ese momento. Por último, hay cuestiones que no se pueden resolver mediante los métodos anteriores, por lo que se tiene que preguntar a los protagonistas del proyecto. Mediante entrevistas individuales y colegiadas a maestros, equipo directivo, alumnado, madres colaboradoras y asesor se pretendió averiguar aquella información no extraída anteriormente y que se hace necesaria para responder a los objetivos propuestos. El tipo de entrevista que se ha elegido es el de entrevista abierta o no estructurada. De igual forma, se ha administrado un cuestionario de 64 ítems: 11 de naturaleza ilustrativa y 53 ítems de seis opciones de respuesta tipo Likert para conocer las representaciones del profesorado del centro acerca del trabajo por proyectos, de los cuales se han seleccionado 11 ítems para el presente estudio (tabla 2).

En cuanto al tratamiento y el análisis de los datos obtenidos en el estudio se han utilizado procedimientos diferentes en función de la naturaleza de los datos obtenidos. Por un lado, el tratamiento de los datos cuantitativos se ha llevado a cabo a través de métodos descriptivos, utilizando la aplicación IBM® SPSS v21. Se realizó una aproximación inicial a los resultados a través de un análisis univariado, caracterizando cada ítem a través de porcentajes. Por su parte, los datos cualitativos han sido codificados manualmente. El proceso de codificación implicó un análisis minucioso de los datos permitiendo una primera interpretación del proceso realizado, así como el establecimiento de relaciones a partir de la información obtenida.

Tabla 2

Ítems del cuestionario sobre trabajo por proyectos

$\mathbf{N}^{\mathbf{o}} \quad$ Ítem

6 Un proyecto no debe planificarse hay que dejar que evolucione con naturalidad

18 La mejor formación es involucrarse en un proyecto y reflexionar para poco a poco conocerlo mejor

19 Nunca he participado en experiencias de trabajo por proyecto

22 A la familia se le informa para que no se oponga

24 Por lo general faltan recursos y medios adaptados a esta forma de desarrollar el currículum

29 Falta formación en el profesorado

32 Preparar y desarrollar un proyecto me ocupa demasiado tiempo personal

$37 \mathrm{El}$ asesoramiento contribuye al éxito de la implantación de los proyectos de trabajo en el aula

39 El trabajo por proyectos motiva al alumnado

41 Participar en experiencias de trabajo por proyecto promueve la formación del profesorado

52 Me gustaría aplicar los proyectos como propuesta didáctica durante todo el curso

\subsection{Caracterización del caso}

La experiencia se desarrolló en un centro de Educación Infantil y Primaria ubicado en una barriada obrera de Huelva capital. El centro contaba con dos líneas desde infantil hasta sexto de primaria y 31 docentes, de los cuales un $60 \%$ eran definitivos y el resto provisionales. En el centro se encontraba escolarizado un $30 \%$ de alumnado cuyos familiares tenían procedencia de países como Argelia, Brasil, Colombia, Marruecos, Ecuador, Nigeria, Polonia y Rumania, además de un 10\% de alumnado gitano. Asimismo, se contabilizaban un gran número de familias desestructuradas por diferentes motivos: abandono familiar, problemas de separación, malos tratos, etc. Por su parte, en los últimos años se han acentuado las situaciones de desempleo, dando como resultado familias en riesgo de exclusión social extrema (análisis de documentos: proyecto de centro; entrevista con Jefa de Estudios). 
Ante una realidad social compleja, tanto el equipo directivo como el claustro en su conjunto consideraron la necesidad de mejorar los procesos de enseñanza/ aprendizaje. Además, los resultados que estaban obteniendo en los últimos cursos escolares hacían pertinente explorar otras alternativas curriculares. Por ello, de la mano de la asesoría de referencia del Centro del Profesorado (CEP) de Huelva, decidieron consolidar una formación en centro sobre trabajo por proyectos con apoyo de casi el 100\% claustro. El proceso se desarrolló a lo largo del curso 2018/2019 con sesiones formativas y de trabajo a lo largo de los meses de octubre y mayo (tabla 3).

Tabla 3

Itinerario formativo

\begin{tabular}{|c|c|c|}
\hline $\begin{array}{l}\text { Tipo de } \\
\text { sesión }\end{array}$ & Mes & Contenido abordado \\
\hline $\begin{array}{l}\text { Formación en } \\
\text { CEP }\end{array}$ & Octubre & Ponencia sobre fundamentos del trabajo por proyectos \\
\hline $\begin{array}{l}\text { Formación en } \\
\text { el centro }\end{array}$ & Noviembre & Análisis de proyectos publicados por parte del equipo docente \\
\hline $\begin{array}{l}\text { Formación en } \\
\text { el centro }\end{array}$ & Diciembre & $\begin{array}{l}\text { Ponencia sobre elementos constitutivos de un proyecto de trabajo y } \\
\text { realización de prácticas }\end{array}$ \\
\hline $\begin{array}{l}\text { Formación en } \\
\text { el centro }\end{array}$ & Enero & $\begin{array}{l}\text {-Reunión de equipo para selección de la temática de estudio y mapa } \\
\text { conceptual } \\
\text {-Reunión de equipo para diseño de red de Preguntas-Problema y } \\
\text { actividades del proyecto }\end{array}$ \\
\hline $\begin{array}{l}\text { Formación en } \\
\text { el centro }\end{array}$ & Febrero & Sesiones de trabajo para la concreción de las actividades del proyecto \\
\hline $\begin{array}{l}\text { Formación en } \\
\text { el centro }\end{array}$ & $\begin{array}{l}\text { Marzo- } \\
\text { abril }\end{array}$ & $\begin{array}{l}\text { Sesiones de trabajo para la finalización de la planificación del proyecto y } \\
\text { elaboración de materiales curriculares }\end{array}$ \\
\hline $\begin{array}{l}\text { Formación en } \\
\text { el centro }\end{array}$ & Mayo & Implementación del proyecto en el aula \\
\hline $\begin{array}{l}\text { Sesión de } \\
\text { trabajo en CEP }\end{array}$ & Mayo & $\begin{array}{l}\text { Sesión final de puesta en común de la experiencia en CEP de Huelva. } \\
\text { Exposición de póster de la experiencia en las II Jornadas de Buenas } \\
\text { Prácticas del CEP de Huelva-Isla Cristina }\end{array}$ \\
\hline
\end{tabular}

Así pues, el profesorado del centro asistió a ponencias, realizó lecturas especializadas, desarrolló dinámicas grupales reflexivas y cumplimentó memorias de progreso y finales; además, colaboró en la elaboración y desarrollo del proyecto de trabajo. Los resultados obtenidos se compartieron en una sesión de trabajo con otros centros educativos en los que se habían desarrollado experiencias formativas en torno a proyectos de trabajo, así como en las II Jornadas de Buenas Prácticas del CEP de Huelva-Isla Cristina.

\section{Análisis y resultados}

3.1. Categoría 1: representaciones del profesorado en torno al itinerario formativo sobre trabajo por proyectos

La primera categoría aborda las concepciones del profesorado en torno al itinerario formativo de trabajo por proyectos, adentrándose también en sus concepciones acerca de la perspectiva educativa. El proceso formativo se generó tras la toma de contacto de la asesoría de referencia del CEP con el colegio: "Cuando me incorporé como asesor de formación al centro vi predisposición a la formación en trabajo por proyectos por parte del equipo directivo" (Entrevista Asesor, p. 4). El estado de opinión proclive a la 
perspectiva educativa era también manifiesto en la docente que asumió la coordinación del proyecto de formación en el centro: "Soy totalmente favorable. La escuela tiene que cambiar porque lo que estamos haciendo no transforma la realidad, no está sirviendo para nada" (Entrevista Coordinadora, p. 1). Por su parte, el 84\% del profesorado del claustro expresaba la expectativa de trabajar por proyectos durante todo el curso (Cuestionario proyectos, ítem 52).

En el centro, sin embargo, no existía una trayectoria generalizada de trabajo por proyectos, con un $50 \%$ del claustro que nunca había participado en una experiencia (Cuestionario proyectos, ítem 19), tal y como manifestaba el siguiente docente: "Mi formación ha sido el curso del CEP (...) no había participado antes en un proyecto de trabajo" (Entrevista Docente, p. 2). Por su parte, el 83\% del profesorado expresaba carencias formativas en trabajo por proyectos (Cuestionario proyectos, ítem 29); al tiempo que el 89\% consideraba necesario involucrarse en un proyecto para conocer la potencialidad de la perspectiva educativa (Cuestionario proyectos, ítem 18).

Tomando en cuenta el compromiso colectivo de embarcarse en un proceso formativo que desembocara en el diseño y desarrollo de un proyecto de trabajo, se organizaron ponencias sobre los fundamentos del trabajo por proyectos y diferentes sesiones de trabajo: "He asistido durante este curso a todas y cada una de las sesiones propuestas tanto en el colegio como en el CEP" (Memoria de seguimiento, Jefa de estudios, p. 5). El proceso desarrollado a lo largo de varios meses permitió, en palabras de uno de los docentes: "llevar a la práctica otros métodos y formas de trabajo que nos abren una nueva y rica perspectiva didáctica" (Memoria final Docente 3, p. 4). La coordinadora del proyecto valoraba el haber iniciado "un camino que espero podamos continuar, una nueva forma de abordar los aprendizajes más contextualizados a la realidad en la que trabajamos y más significativos para nuestro alumnado" (Memoria final Coordinadora, p. 5).

El proceso formativo se vio facilitado por la predisposición del equipo docente a involucrarse en una formación en centro: "Se ha implicado todo el centro, tan solo dos compañeros no han participado en el curso, sin embargo, sí se han implicado en el proyecto ahora cuando lo hemos trabajado con el alumnado" (Entrevista Docente, p. 2), valoración que recalcaba la Jefa de Estudios: "Ha participado todo el mundo creando actividades y las fichas correspondientes... creo que ha sido una participación equitativa" (Entrevista Jefa de estudios, p. 3). Asimismo, se ponía en valor el papel asumido por la asesoría de referencia del centro: "Nos ha conducido por las diferentes fases del proyecto y se ha implicado en nuestro centro desempeñando un liderazgo pedagógico que no lo habíamos visto antes en ningún asesor" (Memoria final Director, p. 7), valorando a su vez el aporte de "muchas ideas y mucha experiencia" (Entrevista Docente, p. 2). Los datos del cuestionario permiten refrendar estas valoraciones, con un 89,5\% del claustro que coincidía en valorar la contribución del asesoramiento al éxito de la implantación de los proyectos de trabajo en el aula (Cuestionario proyectos, ítem 37).

Sin embargo, el tiempo dedicado a las sesiones de formación dificultó “que no se trataran otros temas que normalmente se desarrollan en los ciclos" (Memoria final Director, p. 9). Además del tiempo invertido, se mencionaba como dificultad la dedicación de "mucha energía a conocer los aspectos prácticos, organizativos y metodológicos del trabajo por proyectos” (Memoria final Coordinadora, p. 8). Asimismo, el establecimiento de los fundamentos de la perspectiva educativa de trabajo por proyectos requirió de diferentes sesiones formativas que afectaron el tiempo disponible para las tareas de diseño del proyecto: "Cuando nos dimos cuenta se nos había venido el tiempo encima y estábamos en el diseño de las actividades" (Entrevista Asesor, p. 5). A pesar de estos esfuerzos, el proceso formativo quedó incompleto al no poder abordar con profundidad las cuestiones referentes a la evaluación: "El aspecto de la evaluación no lo hemos abordado con precisión y creo que sería un aspecto a mejorar para el próximo (Memoria final Docente 9, p. 13). En todo caso, se evidenciaron valoraciones positivas del itinerario formativo: "La formación nos ha aportado la información para poder poner en práctica este proyecto" (Memoria final Docente 16, p. 15), logrando que se pusiera en valor el potencial de la perspectiva educativa: "He visto que si se le puede sacar partido al tema de los proyectos" (Entrevista Docente, p. 2). Por su parte, el 94.5\% del profesorado del claustro consideraba que su participación en experiencias de trabajo por proyectos ha ejercido un efecto positivo en su formación (Cuestionario proyectos, ítem 41).

El análisis de la primera categoría pone de manifiesto que el centro educativo partía de una buena predisposición a involucrarse en un proceso formativo en torno al trabajo por proyectos. Además, el compromiso del equipo directivo, claustro y asesoría de referencia contribuyeron al desarrollo del itinerario que permitió el diseño y desarrollo del proyecto, a pesar de advertir algunos obstáculos relacionados con la gestión del tiempo y la evaluación.

\subsection{Categoría 2: diseño y desarrollo de un proyecto de trabajo}


La segunda categoría se ocupa de analizar los procesos de planificación e implementación del proyecto de trabajo. En torno al 63\% de los docentes participantes consideraban que un proyecto no debe planificarse y hay que dejar que evolucione con naturalidad (Cuestionario proyectos, ítem 6). No obstante, esta fase es fundamental para que el posterior desarrollo sea satisfactorio y se alcancen los objetivos que quedarán definidos en este mismo apartado. El proceso de planificación se produjo de forma conjunta en sesiones de trabajo. En primer lugar, se seleccionó la temática del proyecto, la alimentación, tras un debate en el que se justificó su pertinencia en el contexto del centro: "Este proyecto surge por la preocupación que tenemos ante la alimentación de nuestro alumnado en el recreo (...) así como por el consumo de chucherías tanto en el aula como en su vida diaria" (Memoria final Docente 6, p. 7). Seguidamente, se desarrollaron sesiones de trabajo para la realización del análisis didáctico y de contenido. Una vez realizado este proceso se determinó el objeto de estudio, que dio título al proyecto: ¿Qué tenemos que comer para estar sanos y sanas? y se concretaron las preguntas-problema, contenidos y actividades del proyecto.

\section{Tabla 4}

Preguntas-problema, contenidos y actividades del proyecto

Preguntas-Problema Contenidos Actividades

1. ¿Qué comemos? Análisis de la dieta

-Mural "Lo que sabemos, queremos saber y lo que vamos aprendiendo sobre la alimentación"

-Los sentidos a examen

-Investigamos las golosinas.

$\begin{array}{lll}\text { 2. ¿Qué nos aportan } & \text { Composición de los alimentos } & \text {-Taller Ensaladas con arte } \\ \text { los alimentos? } & & \text {-Investigando las etiquetas }\end{array}$

3. ¿Sabemos conservar los alimentos?

Etiquetado, caducidad y conservación de los alimentos

Cultura gastronómica

4. ¿En todos los sitios se come lo mismo?

5. ¿Puedo enfermar con lo que como?
Higiene y manipulación de alimentos
-Construimos la pirámide de los alimentos

-Salida al supermercado

-Taller de cocina: degustación

-Taller de limpieza de manos

A partir de la identificación de las preguntas-problema se diseñaron actividades con la finalidad de darles respuesta: "tales como salidas al mercado, murales, experimentos, actividades grupales y de elaboración de recetas, etc. (Memoria final Docente 3, p. 3). Aunque finalmente la propuesta fue considerada excesivamente ambiciosa: "Hemos querido abarcar muchos aspectos en este proyecto. Deberíamos haber elegido menos actividades y aplicarlas mejor en la práctica" (Memoria final Jefa de Estudios, p. 6). Por otra parte, las tareas de planificación hicieron necesaria la coordinación entre el profesorado de las diferentes áreas, aspecto que supone un reto en un contexto educativo caracterizado por el trabajo individual: "realizar un proyecto es una tarea muy compleja y se necesita mucho más tiempo para poder coordinarse bien con los compañeros" (Memoria final Docente 10, p. 9). Refutando el argumento anterior, más del $89 \%$ de los docentes coincidían en que preparar y desarrollar un proyecto les ocupa demasiado tiempo personal (Cuestionario proyectos, ítem 32).

Los recursos y materiales que se han utilizado provienen del reciclaje, de la propia elaboración del alumnado o de una ayuda externa: "muchos de los recursos los ha aportado el centro ya que estamos en una zona desfavorecida" (Entrevista 1, p. 3). Un 89\% del claustro de profesorado demanda la falta de recursos y medios adaptados para el desarrollo del trabajo por proyectos en el centro (Cuestionario proyectos, ítem 
24). No obstante, la calidad y cantidad de recursos, en su inmensa mayoría va a depender de la imaginación y creatividad del docente. Por último, la evaluación de un proyecto creó incertidumbre en el profesorado: "¿Cómo evalúo yo la actividad que hemos hecho hoy? porque todos lo han hecho y han participado" (Entrevista 1, p. 1), manifestándose su insuficiente cobertura en el proceso formativo: "La evaluación no la hemos abordado concienzudamente en nuestro ciclo" (Memoria final docente 6, p. 7).

Analizando el papel del profesorado en la implementación del proyecto se puso de relieve la necesidad de contar con recursos humanos complementarios para la elaboración de determinadas actividades. Específicamente, se reclamó la figura de un coordinador de proyectos interno en el centro para la dinamización de este tipo de iniciativas ante el alto número de responsabilidades: "ha faltado la figura de un coordinador que nos facilitara la sincronía en las actividades. Los tutores carecemos de tiempo (...) por lo que a veces nos sentimos desbordados por tanta tarea" (Memoria final Docente 10, p. 9).

En cuanto al alumnado, el proyecto tuvo una acogida muy satisfactoria: "el logro que ha sido más notable es el interés y motivación por parte del alumnado a la hora de realizar las distintas actividades y experiencias del proyecto" (Memoria final Docente 3, p. 3). La motivación que experimenta el alumnado aumenta durante el desarrollo de proyectos, como así atestigua el 100\% del claustro (Cuestionario proyectos, ítem 39). Asimismo, se valora que el alumnado haya comenzado a "cuestionarse lo que comen y a decidir lo que es bueno para su salud" (Memoria final Docente 11, p. 10). Son muchas las alusiones a las mejoras: "Ver la cara de los discentes cuando ellos mismos descubren la cantidad de azúcar que tiene una lata de Coca-Cola, y escuchar cómo se replantean beber otro tipo de líquidos sin azúcar, es muy satisfactorio" (Memoria final Docente 9, p. 8). De este modo, se han producido mejoras significativas en "el consumo de frutas que se toman ahora en la hora del recreo y modificación en la concepción de los hábitos sanos relacionados con la alimentación" (Memoria final Docente 9, p. 9).

El profesorado del centro era consciente de que el cambio metodológico iniciado podía generar desconfianza e incertidumbre en la familia del alumnado. Por ello, más del 68\% de los docentes consideraban necesario informar a las familias para que no se opusieran a la implementación del proyecto en el aula (Cuestionario proyectos, ítem 22). Aun estando informadas, la participación familiar fue percibida como escasa por parte de los docentes participantes: "Para mí, la dificultad más destacada es la poca colaboración que encontramos en las familias, creo que el proyecto se podría haber enriquecido mucho más con su colaboración" (Memoria final docente 16, p. 13).

$\mathrm{El}$ análisis de los elementos clave de la segunda categoría en torno a la planificación del proyecto y su implementación revela un escenario favorable al trabajo por proyectos, si bien son necesarios ajustes y cambios en el funcionamiento del centro para hacer de esta forma de trabajar una apuesta sostenible y consolidada en el tiempo.

\section{Discusión y conclusiones}

Con la finalidad de garantizar la máxima claridad expositiva analizaremos cada uno de los objetivos del estudio en función de los resultados obtenidos.

El primer objetivo que nos planteamos en la investigación consistía en indagar acerca de las representaciones del profesorado en torno al desarrollo del proceso formativo de trabajo por proyectos.

La propuesta de participación en el itinerario formativo fue de naturaleza exógena, planteada en su origen por la asesoría de referencia; sin embargo, tanto el equipo directivo como el profesorado del centro se mostraron altamente receptivos a comprometerse con el proceso desde su inicio. A pesar de que alrededor de la mitad del claustro no tenía experiencia ni formación previa en proyectos, las representaciones docentes en torno a la temática eran altamente positivas, siendo mayoritaria la predisposición a diseñar y desarrollar experiencias. Precisamente, las concepciones docentes favorables al trabajo por proyectos y sus beneficios para el alumnado han demostrado ser más determinantes que la propia formación previa a la hora de propiciar la participación del profesorado (Tamim y Grant, 2013).

El proceso formativo exigió un alto nivel de compromiso y exigencia para el conjunto del claustro y la asesoría de formación, quienes dedicaron tiempo y esfuerzos al desarrollo de las actuaciones formativas propuestas. La implicación del profesorado fue mayoritaria en un proceso que se alargó durante todo un curso escolar. Al final del periodo formativo, el profesorado accedió a los fundamentos de los proyectos de trabajo, así como a técnicas y estrategias para su diseño e implementación práctica. En este sentido, el análisis de sus declaraciones evidencia una evolución en su desarrollo profesional a partir de la modalidad de formación vinculada a un proyecto de centro, así como lo han evidenciado diferentes estudios en experiencias previas (Escudero et al., 2017; Seijo et al., 2015; Torrego et al., 2015). 
No obstante, el desarrollo del itinerario formativo redujo la disponibilidad de tiempo para la realización de otras tareas inherentes a su práctica profesional, aspecto ampliamente identificado en otras experiencias de proyectos (Iglesias et al., 2018; Rodríguez-Miranda et al., 2012). Este hecho no mermó la predisposición del profesorado a continuar su formación para el siguiente curso, demandando a su vez un abordaje específico de la evaluación.

El segundo objetivo perseguía analizar las concepciones y prácticas del profesorado en torno al diseño y desarrollo de un proyecto de trabajo.

El proyecto requirió la implicación del profesorado en múltiples tareas de corte curricular: desde la celebración de reuniones de coordinación y la elaboración de materiales, a la toma de decisiones en torno a las actividades y demás elementos curriculares. En este sentido, se experimentaron cambios en la cultura de colaboración del profesorado, trascendiendo a las interacciones de corte técnico para adentrarse en la gestación de debates pedagógicos enfocados a la mejora de los procesos de enseñanza/aprendizaje.

Por su parte, el profesorado valoró muy positivamente la incidencia del proyecto en el alumnado, destacando su implicación y motivación en el desarrollo de las actividades; conclusión similar a la obtenida en múltiples estudios sobre la implementación de proyectos (Basilotta et al, 2017; Botella y Ramos, 2020). Llama la atención la discrepancia existente entre la motivación que expresa el profesorado por participar en propuestas innovadoras, puesta de manifiesto en este estudio, así como en otros (Martínez et al., 2018), con una realidad educativa en la que los enfoques transmisivos siguen imperando. Siguen existiendo, por tanto, obstáculos de gran calado que han de ser superados si queremos alcanzar escenarios educativos donde las metodologías activas sean una realidad generalizada en los centros.

Asimismo, la escasa participación de las familias y, en ocasiones, la falta de recursos necesarios para la implementación del proyecto, fueron dos obstáculos que el profesorado tuvo que afrontar. En Cañal et al. (2011) se analizan estos y otros obstáculos que han de ser superados para el éxito de las experiencias.

A partir del estudio realizado se constata que el diseño y desarrollo de un proceso formativo para fomentar el trabajo por proyectos en un centro con unas características comunes a otros muchos, no está exento de dificultades, pero a su vez aporta importantes ventajas tanto para el profesorado como para su alumnado. Para que estas iniciativas sean exitosas y sostenibles en el tiempo proponemos finalmente una serie de aspectos a considerar, a saber:

Insistencia en la necesidad del cambio de paradigma educativo. La formación en trabajo por proyectos debe conjugar las cuestiones técnicas con las de fundamentación epistemológica, apostando por "un cambio de paradigma en la forma del pensar del profesorado: educamos como nos han educado a nosotros" (Arias, 2017, p. 65). El reto consiste en despertar en el profesorado un cambio de mirada en torno a la finalidad del trabajo educativo que le permita desafiar las estructuras tradicionales, a la vez que ofrecerle herramientas y estrategias útiles (Zuniga y Cooper, 2016). Agregando a lo anterior, han de crearse marcos burocratizadores favorables al trabajo por proyectos, inexistentes a día de hoy (Beane, 2005), por ejemplo, a través de disponer de exoneraciones horarias para el profesorado encargado de coordinar y dinamizar proyectos.

Potenciación de la figura del docente como investigador. Para llevar a cabo proyectos es imprescindible que el docente asuma la figura de investigador, acompañando al alumnado en el viaje-aventura y compartiendo las incertidumbres que puedan aparecer (Carbonell, 2015). En ese proceso tiene que ser capaz de "aprender a desaprender", dejar atrás ataduras y combatir la zona de confort. Solo llevando a cabo una continua actualización de saberes, se pueden alcanzar los objetivos y, lo que es más importante, lograr la sostenibilidad en el tiempo (López Martín, 2019).

Evitar la sobredimensión de los proyectos acotando su planificación. Una tendencia observada en la planificación de los proyectos se refiere bien a la realización de diseños excesivamente amplios en los que se multiplican los objetivos y las actividades, o al "formalismo excesivo" al que alude Pozuelos (2007). Ambas prácticas repercuten en los procesos de diseño, desarrollo y evaluación de los proyectos, pudiendo comprometer la sostenibilidad futura de las experiencias en los centros al contribuir a la sensación de sobrecarga en el profesorado.

Distribuir responsabilidades dentro de una cultura de la participación e identificar aliados clave. El diseño y desarrollo de un proyecto de trabajo exitoso es una labor colegiada, en la que han de participar diferentes personas que la hagan posible. Los proyectos unipersonales pueden llegar a ser exitosos, pero se compromete su sostenibilidad ya que trabajar por proyectos implica generalmente aumentar el tiempo de planificación (Aksela \& Haatainen, 2019). A su vez, en el momento en que un equipo de trabajo docente comienza a planificar un proyecto se han de distribuir responsabilidades que permitan enriquecerlo sin agotar a ninguno de sus miembros. Las familias pueden llegar a ser aliados clave en los procesos de diseño e implementación de los proyectos, una vez son conscientes de los beneficios que esta perspectiva educativa ofrece a sus hijos/as (Robinson y Aronica, 2015). 
Creación de un banco de proyectos en el centro: hacia la sostenibilidad en el tiempo. El gran reto del trabajo por proyectos es su pervivencia y arraigo en los centros educativos. Trascender a los modelos de proyectos episódicos sigue representando una tarea pendiente ante la que no se ha logrado dar respuestas definitivas. Sin embargo, la creación de bancos de proyectos en los centros permitiría contar con un patrimonio didáctico que evitaría el eterno comienzo desde cero y las consecuencias que esto conlleva. Estudios previos en formación del profesorado han evidenciado las ventajas de que el profesorado cuente con los recursos y ejemplificaciones de proyectos previamente desarrollados en la práctica (Domènech-Casal et al., 2019), de especial relevancia para el profesorado de nueva incorporación a los centros.

Analizando por último las limitaciones encontradas en el desarrollo de la investigación, es preciso advertir que el desarrollo de un estudio de casos único condiciona la transferencia de las conclusiones al analizar un marco de referencia muy concreto y local. Por ello, son pertinentes estudios que engloben un volumen mayor de casos con la finalidad de conocer cuáles son los elementos clave que limitan y posibilitan el desarrollo de proyectos de trabajo en el aula. También, sería preciso seguir profundizando en las diferentes modalidades formativas y su capacidad para fomentar el desarrollo profesional docente en el marco de la integración curricular y el aprendizaje significativo del alumnado. Los cambios sociales y los retos de las sociedades complejas en las que vivimos demandan alternativas curriculares capaces de despertar el sentido crítico, la comprensión, transformación y mejora de los contextos en los que vive y se desarrolla el alumnado. La perspectiva educativa de trabajo por proyectos representa hoy más que nunca una referencia, que lejos de ofrecer todas las respuestas, tiene la potencialidad de superar los modelos basados en la pedagogía de la indiferencia (Lingard, 2007) y así generar las preguntas necesarias y, en suma, abrir la escuela a la vida.

\section{Apoyos}

Financiado por el proyecto I $+\mathrm{D}+\mathrm{i}$ “Anatomía del cambio educativo: las escuelas ante el reto de la innovación pedagógica" (UHU-1256182).

\section{Referencias}

Aksela, M., \& Haatainen, O. (2019). Project-Based Learning (PBL) in Practise: Active Teachers' Views of Its Advantages and Challenges. In Integrated Education for the Real World: 5th International STEM in Education Conference Post-Conference Proceedings. Queensland University of Technology, 9-16, International STEM in Education Conference, Brisbane, Australia.https://bit.ly/3obSfGm

Arias, L. (2017). El aprendizaje por proyectos: una experiencia pedagógica para la construcción de espacios de aprendizaje dentro y fuera del aula. Ensayos Pedagógicos, 12(1), 5168. https://doi.org/10.15359/rep.12-1.3

Basilotta, V., Martín del Pozo, M. \& García-Valcárcel, A. (2017). Project-based learning (PBL) through the incorporation of digital technologies: An evaluation based on the experience of serving teachers. Computers in Human Behavior, 68, 501-512. https://doi.org/10.1016/j.chb.2016.11.056

Beane, J. A. (2005). Integración del curriculum. Morata.

Botella, A. M. y Ramos, P. (2020). Motivación y Aprendizaje Basado en Proyectos: una Investigación-Acción en Educación Secundaria. Multidisciplinary Journal of Educational Research, 10(3), 295-320. http://dx.doi.org/10.17583/remie.2020.4493

Cañal, P., Travé, G. y Pozuelos, F. J. (2011). Análisis de obstáculos y dificultades de profesores y estudiantes en la utilización de enfoques de investigación escolar. Investigación en la Escuela, 73, 5-26. https://doi.org/10.12795/IE.2011.i73.01

Carbonell, J. (2015). Pedagogias del siglo XXI: alternativas para la innovación educativa. Ediciones Octaedro.

Chen, C. H. \& Yang, Y. C. (2019). Revisiting the effects of project-based learning on students' academic achievement: A meta-analysis investigating moderators. Educational Research Review, 26, 71-81. https://doi.org/10.1016/i.edurev.2018.11.001

Domènech-Casal, J., Lope, S., y Mora, L. (2019). Qué proyectos STEM diseña y qué dificultades expresa el profesorado de secundaria sobre Aprendizaje Basado en Proyectos. Revista Eureka sobre Enseñanza y $\begin{array}{lllll}\text { Divulgación de las } & \text { Ciencias, } & \text { 16(2), }\end{array}$ http://dx.doi.org/10.25267/Rev Eureka ensen divulg cienc.2019.v16.i2.2203

Escudero, J. M., Cutanda, M. T. y Trillo, J. F. (2017). Aprendizaje docente y desarrollo profesional del profesorado. Profesorado. Revista de Currículum y formación de profesorado, 21(3), 83-102. https://bit.ly/3iGgw6k

Gutiérrez, R. (2015). El trabajo por proyectos para la construcción compartida del currículum de artes plásticas y visuales en la formación del profesorado de Educación Infantil. ENSAYOS, Revista de la Facultad de Educación de Albacete, 30(2). https://bit.ly/3unzrs5 
González-Rodríguez, A., Travé, G. (H). y García Padilla, F. M. (2020). La educación nutricional a partir del trabajo por proyectos en Educación Primaria. Didáctica de las ciencias experimentales y sociales, 38, 171-186. https://doi.org/10.7203/dces.38.15376

Guo, S. \& Yang, Y. (2012). Project-based learning: an effective approach to link teacher professional development and students learning. Journal of Educational Technology Development and Exchange, 5(2), 4156. https://doi.org/10.18785/jetde.0502.04

Han, S. Y., Yalvac, B., Capraro, M. M. \& Capraro, R. M. (2015). In-service Teachers' Implementation and Understanding of STEM Project Based Learning. Eurasia Journal of Mathematics, Science and Technology Education 11(1), 63-76. https://doi.org/10.12973/eurasia.2015.1306a

Halinen, I. (2018). The new educational curriculum in Finland. Improving the quality of childhood in Europe, 7, 75-89. https://bit.ly/3o9D7cp

Kokotsaki, D., Menzies,V. \& Wiggins, A. (2016). Project-based learning: A review of the literature. Improving Schools, 19(3), 267-277. https://doi.org/10.1177/1365480216659733

Iglesias, M. J., Lozano, I. y Roldán, I. (2018). La calidad e innovación educativa en la formación continua docente: un estudio cualitativo en dos centros educativos. Revista Iberoamericana de Educación, 77(1), 1334. https://doi.org/10.35362/rie7713090

Larmer, J. \& Mergendoller, J. R. (2010). 7 Essentials for Project-Based Learning. Educational Leadership, 68(1), 34-37. https://bit.ly/37QfVup

Leat, D. (Ed.). (2017). Enquiry and project based learning: Students, school and society. Taylor \& Francis.

Lingard, B. (2007). Pedagogies of indifference. International Journal of Inclusive Education, 11(3), 245-266. https://doi.org/10.1080/13603110701237498

López Martín, R. (2019). La innovación docente en la formación del profesorado. Principios y directrices de futuro. Revista Crónica, 4, 83-94. https://bit.ly/37GZBfq

Majó, F. y Baqueró, M. (2014). Los proyectos interdisciplinarios. Graó.

Martínez, M. J. I., Cabezas, I. L. y Soler, I. R. (2018). La calidad e innovación educativa en la formación continua docente: un estudio cualitativo en dos centros educativos. Revista Iberoamericana de Educación, 77(1), 13-34. https://doi.org/10.35362/rie7713090

Pozuelos, F. J. (2007). Trabajos porproyectos en el aula: descripción, investigación y experiencias. Publicaciones MCEP.

Pozuelos, F. J., García-Prieto, F. J. y Gracia-Rivera, F. J. (2017). Trabajo por proyectos para la promoción de la alimentación en la escuela en el medio rural. Aula de innovación educativa, 262, 43-48. https://bit.ly/2Mmn0LZ

Pozuelos, F. J. y García-Prieto, F. J. (2020). Currículum integrado: estrategias para la práctica. Investigación en la Escuela, 100, 37-54. https://doi.org/0.12795/IE.2020.1100.04

Robinson, K y Aronica, L. (2015). Escuelas creativas: La revolución que está transformando la educación. Penguin.

Rodríguez-Miranda, F. P., Pozuelos, F. J y García-Prieto, F. J. (2012). Cuando el cambio llega a la escuela. Estudio de casos sobre los procesos de innovación educativa. Revista Electrónica de Investigación y Docencia (REID), 8, 117-141. https://bit.ly/3iMlcHN

Sanmarti, N. y Márquez, C. (2017). Aprendizaje de las ciencias basado en proyectos: del contexto a la acción. Ápice, 1(1), 3-16. https://doi.org/10.17979/arec.2017.1.1.2020

Santos Guerra, M. Á. (2010). La formación del profesorado en las instituciones que aprenden. Revista Interuniversitaria de formación del profesorado, 24(2), 175-200. https://bit.ly/3pIBrYk

Sarason, S. (2003). El predecible fracaso de la reforma educativa. Octaedro.

Seijo, J. C. T., López, C. M., López, M. L. P. y Virseda, C. M. (2015). Formación del profesorado en aprendizaje cooperativo y alumnos con altas capacidades: un enfoque inclusivo. Revista latinoamericana de educación inclusiva, 9(2), 91-110. https://bit.ly/3bB0BTB

Soto, A. y Travé, G. (2018). Las representaciones y prácticas docentes acerca de los materiales curriculares. $\begin{array}{lllll}\text { Cambios y } & \text { permanencias. Universitas Psycbologica, 17(5), }\end{array}$ https://doi.org/10.11144/Javeriana.upsy17-5.rpdm

Stake, R. E. (1999). Investigación con estudio de casos. Morata.

Stenhouse, L. (1987). La investigación como base de la enseñanza. Morata.

Tamim, S. R. \& Grant, M. M. (2013). Definitions and uses: Case study of teachers implementing projectbased learning. Interdisciplinary Journal of Problem-based Learning, 7(2), 72-101. https://doi.org/10.7771/1541-5015.1323

Torrego, J. C., Monge, C., Pedrajas, M. y Martínez, C. (2015). Formación del profesorado en aprendizaje cooperativo y alumnos con altas capacidades. Revista latinoamericana de educación inclusiva, 9(2), 91-110. https://bit.ly/3dIP3QQ

Travé, G. (H)., Pozuelos F. J. y Travé, G. (2019). El trabajo por proyectos como alternativa metodológica para la integración lingüística y curricular. Estudio de un caso en un centro bilingüe. Profesorado, Revista 
de Curriculum y Formación del Profesorado, 23(1), 421-440.

https://doi.org/10.30827/profesorado.v23i1.9161

Zuniga, A. \& Cooper, T. M. (2016). Project-Based Learning: 7 Ways to Make It Work. Educational Leadership, 73, 72-76. https://bit.ly/3aV21ZW 